\title{
WEIGHTED BOUNDEDNESS OF THE HARDY-LITTLEWOOD MAXIMAL AND CALDERÓN-ZYGMUND OPERATORS ON ORLICZ-MORREY AND WEAK ORLICZ-MORREY SPACES
}

\author{
RYOTA KAWASUMI AND EIICHI NAKAI*
}

\begin{abstract}
For the Hardy-Littlewood maximal and Calderón-Zygmund operators, the weighted boundedness on the Lebesgue spaces are well known. We extend these to the Orlicz-Morrey spaces. Moreover, we prove the weighted boundedness on the weak Orlicz-Morrey spaces. To do this we show the weak-weak modular inequality. The Orlicz-Morrey space and its weak version contain weighted Orlicz, Morrey and Lebesgue spaces and their weak versions as special cases. Then we also get the boundedness for these function spaces as corollaries.
\end{abstract}

Mathematics subject classification (2020): 46E30, 42B35, 42B25, 42B20.

Keywords and phrases: Orlicz-Morrey space, modular inequality, maximal function, singular integral.

\section{REFERENCES}

[1] R. R. Colfman, Distribution function inequalities for singular integrals, Proc. Nat. Acad. Sci. U.S.A. 69 (1972), 2838-2839.

[2] R. R. COIFMAN AND C. FEFFERMAN, Weighted norm inequalities for maximal functions and singular integrals, Studia Math. 51 (1974), 241-250.

[3] G. P. Curbera, J. García-Cuerva, J. M. Martell and C. Pérez, Extrapolation with weights, rearrangement-invariant function spaces, modular inequalities and applications to singular integrals, Adv. Math. 203 (2006), no. 1, 256-318.

[4] F. Deringoz, V.S. Guliyev, E. Nakai, Y. Sawano And M. Shi, Generalized fractional maximal and integral operators on Orlicz and generalized Orlicz-Morrey spaces of the third kind, Positivity 23 (2019), no. 3, 727-757.

[5] F. DeRINGOZ, V.S. GuliYeV AND S. SAMKo, Boundedness of the maximal and singular operators on generalized Orlicz-Morrey spaces, Operator theory, operator algebras and applications, 139-158, Oper. Theory Adv. Appl., 242, Birkhäuser/Springer, Basel, 2014.

[6] X. FU, D. YANG AND W. YUAN, Boundedness of multilinear commutators of Calderón-Zygmund operators on Orlicz spaces over non-homogeneous spaces, Taiwanese J. Math. 16 (2012), no. 6, $2203-$ 2238.

[7] S. Gala, Y. Sawano and H. Tanaka, A remark on two generalized Orlicz-Morrey spaces, J. Approx. Theory 198 (2015), 1-9.

[8] J. García-Cuerva And J. Rubio de Francia, Weighted norm inequalities and related topics, North-Holland Mathematics Studies, 116. Notas de Matemática [Mathematical Notes], 104. NorthHolland Publishing Co., Amsterdam, 1985. x+604 pp. ISBN: 0-444-87804-1.

[9] L. Grafakos, Classical Fourier analysis, Third edition, Graduate Texts in Mathematics, 249, Springer, New York, 2014.

[10] V. S. GuliYev, S. G. Has anov, Y. Sawano and T. NoI, Non-smooth atomic decompositions for generalized Orlicz-Morrey spaces of the third kind, Acta Appl. Math. 145 (2016), no. 1, 133-174.

[11] J. Gustavsson And J. Peetre, Interpolation of Orlicz spaces, Studia Math. 60 (1977), no. 1, $33-$ 59.

[12] K.-P. Ho, Vector-valued maximal inequalities on weighted Orlicz-Morrey spaces, Tokyo J. Math. 36 (2013), no. 2, 499-512. 
[13] K.-P. Ho, Weak type estimates of singular integral operators on Morrey-Banach spaces, Integral Equations Operator Theory 91 (2019), no. 3, Paper no. 20, 18 pp.

[14] K.-P. Ho, Definability of singular integral operators on Morrey-Banach spaces, J. Math. Soc. Japan 72 (2020), no. 1, 155-170.

[15] R. Hunt, B. Muckenhoupt And R. Wheeden, Weighted norm inequalities for the conjugate function and Hilbert transform, Trans. Amer. Math. Soc. 176 (1973), 227-251.

[16] R. Kawasumi and E. Nakai, Pointwise multipliers on weak Orlicz spaces, Hiroshima Math. J. 50 (2020), no. 2, 169-184.

[17] R. KaWASumi, E. NAKai AND M. ShI, Characterization of the boundedness of generalized fractional integral and maximal operators on Orlicz-Morrey and weak Orlicz-Morrey spaces, to appear in Math. Nachr., https://arxiv.org/abs/2107.10553.

[18] H. KiTA, Orlicz spaces and their applications (Japanese), Iwanami Shoten, Publishers. Tokyo, 2009.

[19] V. Kokilashvili and M. KrbeC, Weighted inequalities in Lorentz and Orlicz spaces, World Scientific Publishing Co., Inc., River Edge, NJ, 1991.

[20] Y. Komori-FuruYA, Weak $L^{p}$-boundedness of the Hardy-Littlewood maximal function (in Japanese), Report collection of Harmonic Analysis Seminar 2011 at Osaka University, 39-42, printed in 2012 .

[21] Y. Komori AND S. Shirai, Weighted Morrey spaces and a singular integral operator, Math. Nachr. 282 (2009), no. 2, 219-231.

[22] P. LiU And M. WAng, Weak Orlicz spaces: Some basic properties and their applications to harmonic analysis, Sci. China. Math. 56 (2013), 789-802.

[23] M. A. Krasnoselsky And Y. B. Rutitsk y, Convex functions and Orlicz spaces, Translated from the first Russian edition by Leo F. Boron, P. Noordhoff Ltd., Groningen 1961.

[24] L. Maligranda, Indices and interpolation, Dissertationes Math. (Rozprawy Mat.) 234 (1985), 49 pp.

[25] L. Maligranda, Orlicz spaces and interpolation, Seminars in mathematics 5, Departamento de Matemática, Universidade Estadual de Campinas, Brasil, 1989.

[26] B. Muckenhoupt, Weighted norm inequalities for the Hardy maximal function, Trans. Amer. Math. Soc. 165 (1972), 207-226.

[27] E. NAKAI, Generalized fractional integrals on Orlicz-Morrey spaces, Banach and Function Spaces (Kitakyushu, 2003), Yokohama Publishers, Yokohama, 2004, 323-333.

[28] E. NaKaI, Orlicz-Morrey spaces and the Hardy-Littlewood maximal function, Studia Math. 188 (2008), no. 3, 193-221.

[29] E. NAKAI, Calderón-Zygmund operators on Orlicz-Morrey spaces and modular inequalities, Banach and function spaces II, 393-410, Yokohama Publ., Yokohama, 2008.

[30] R. O'NeIL, Fractional integration in Orlicz spaces. I., Trans. Amer. Math. Soc. 115 (1965), 300-328.

[31] Y. SAWANO, Singular integral operators acting on Orlicz-Morrey spaces of the first kind, Nonlinear Stud. 26 (2019), no. 4, 895-910.

[32] Y. Sawano, S. Sugano And H. TanaKa, Orlicz-Morrey spaces and fractional operators, Potential Anal. 36 (2012), no. 4, 517-556.

[33] M. SHI, R. ARAI AND E. NAKAI, Generalized fractional integral operators and their commutators with functions in generalized Campanato spaces on Orlicz spaces, Taiwanese J. Math. 23 (2019), no. 6, 1339-1364.

[34] M. ShI, R. ARAi AND E. NAKaI, Commutators of integral operators with functions in Campanato spaces on Orlicz-Morrey spaces, Banach J. Math. Anal. 15 (2021), no. 1, Paper no. 22, 41 pp.

[35] A. ToRChins KY, Interpolation of operations and Orlicz classes, Studia Math. 59 (1976), no. 2, 177 207.

[36] K. Yabuta, Generalizations of Calderón-Zygmund operators, Studia Math. 82 (1985), 17-31. 